\title{
Upregulated Glycolysis Correlates With Clinical and Transcriptomic Significances in Head and Neck Squamous Cell Carcinoma
}

Hideyuki Takahashi ( $\nabla$ htakahas@gunma-u.ac.jp )

Gunma University Graduate School of Medicine

\section{Reika Kawabata-Iwakawa}

Gunma University Initiative for Advanced Research

\section{Shota Ida}

Gunma University Graduate School of Medicine

Ikko Mito

Gunma University Graduate School of Medicine

\section{Hiroe Tada}

Gunma University Graduate School of Medicine

\section{Kazuaki Chikamatsu}

Gunma University Graduate School of Medicine

\section{Research Article}

Keywords: Altered metabolism, Cancer cells, facilitate immunosuppression, tumor progression and immunosuppression

Posted Date: April 19th, 2021

DOl: https://doi.org/10.21203/rs.3.rs-421251/v1

License: (9) This work is licensed under a Creative Commons Attribution 4.0 International License. Read Full License 


\section{Abstract}

Altered metabolism is an emerging hallmark of cancer. Cancer cells preferentially utilize glycolysis for energy production, termed "aerobic glycolysis." In this study, we performed a comprehensive analysis of the glycolysis status in the tumor microenvironment (TME) of head and neck squamous cell carcinoma (HNSCC) using data from The Cancer Genome Atlas database. We first divided 520 patients with HNSCC into two groups based on the mRNA expression of 16 glycolysis-related genes. The glycolysis-high signature positively correlated with human papillomavirus-negative tumor type, advanced $\mathrm{T}$ factor, and unfavorable prognosis. The gene set enrichment analysis revealed upregulation of several pathways, including interferon-alpha response, myc targets, hypoxia, epithelial-mesenchymal transition, transforming growth factor- $\beta$ signaling, and interleukin 6-Janus kinase-signal transducer and activator of transcription 3 signaling, in the glycolysis-high group. Immune cell enrichment analysis revealed decreased infiltration of T cells, dendritic cells, and B cells in the glycolysis-high group, suggesting impaired tumor antigen presentation, $T$ cell activation, and antibody production in TME. Moreover, the expression of TGFB1, CD274, and PDCD1LG2, which facilitate immunosuppression in the TME, was upregulated in the glycolysis-high group. Collectively, these findings suggest the potential of glycolysis monitoring as a biomarker for tumor progression and immunosuppression in the TME of HNSCC.

\section{Introduction}

Head and neck squamous cell carcinoma (HNSCC) is the sixth most common cancer worldwide, with a reported incidence of 890,000 new cases and 450,000 deaths annually ${ }^{1,2}$. The major risk factors of HNSCC include exposure to tobacco-derived carcinogens and excessive alcohol consumption ${ }^{3}$. Moreover, prior infection with oncogenic strains of human papillomavirus (HPV) has recently been recognized as a risk factor of oropharyngeal cancers, especially in the USA and Western Europe ${ }^{4}$. As the 5-year survival rate of HNSCC is still $66 \%$, new therapeutic modalities have been explored. Recent breakthroughs in cancer therapy have resulted in novel immunotherapy regimens targeting the immune checkpoint programmed death 1 (PD-1) in patients with $\mathrm{HNSCC}^{5,6}$. However, the survival benefit is limited to only $20 \%-30 \%$ patients, and new biomarkers that predict the efficacy of anti-PD-1 therapy have been investigated. Accumulating evidence has indicated that in HNSCC, tumor cells orchestrate a highly immunosuppressive tumor microenvironment (TME) via the production of immunosuppressive mediators, recruitment of various stromal cells, expression of immune checkpoint ligands, and downregulation of human leukocyte antigen expression ${ }^{7,8}$, which is referred to as immune evasion ${ }^{9}$. As immune checkpoint inhibitors, including anti-PD-1 antibodies, target the interaction between tumor cells and T cells, a comprehensive understanding of complex immune networks in the TME is needed to establish a new biomarker for immunotherapies.

Reprogramming of energy metabolism is an emerging hallmark of cancers ${ }^{10,11}$. Even during aerobic conditions, cancer cells preferentially utilize glycolysis for producing energy, termed "aerobic glycolysis"12,13. Increased glycolysis correlates with aggressive tumor progression, treatment resistance, 
and unfavorable prognosis in various cancers, including HNSCC ${ }^{14-17}$. The upregulation of glucose transporter-1 (GLUT1), which transports glucose into the cytoplasm, increases glucose utilization and is associated with aggressive behavior in several cancers ${ }^{18}$. Increased glucose uptake via GLUT1 has been investigated in clinics using positron emission tomography, which monitors the uptake of a radiolabeled glucose analog, ${ }^{18} \mathrm{~F}$-fluorodeoxyglucose. Moreover, both oncogenic signaling pathways and hypoxia induce aerobic glycolysis in cancer cells. Activation of oncogenes such as RAS, MYC, and MAPK and PI3K-AKT signaling as well as TP53 mutation, a tumor suppressor gene, are associated with increased

glycolysis ${ }^{19-23}$. The hypoxic TME also alters metabolic reactions in tumor cells by upregulating the expression of glucose transporters and enzymes of the glycolytic pathway, including transcription factors, hypoxia-inducible factor (HIF) $1 \mathrm{a}$ and HIF2 $\mathrm{a}^{24-26}$. However, the primary mechanism that regulates glycolysis in cancer cells still remains unclear. Furthermore, the relationship between upregulated glycolysis and clinicopathological features is elusive in various cancer types. Notably, a few studies have revealed the interplay between tumor glycolysis and immune evasion ${ }^{15,27,28}$. A comprehensive analysis that focuses on the relationship between glycolysis and immunological significance in the TME is warranted.

In the present study, we performed a comprehensive analysis of glycolysis in HNSCC by analyzing transcriptome and clinical data regarding HNSCC obtained from The Cancer Genome Atlas (TCGA) database. Based on the mRNA expression of 16 glycolysis-related genes, we segregated patients with HNSCC into two groups and performed a comprehensive analysis to compare the clinical and biological significance between the glycolysis-high and glycolysis-low groups.

\section{Results}

\section{Glycolysis-high gene signature positively correlated with locally advanced tumors and unfavorable prognosis}

We first performed non-supervised hierarchical clustering of 520 patients with HNSCC based on data on the expression of 16 glycolysis-related genes obtained from TCGA database (Figure 1a). Then, patients were divided into two groups; both groups were compared in terms of clinical parameters, including HPV status, primary lesion, $\mathrm{T}$ factor, $\mathrm{N}$ factor, $\mathrm{M}$ factor, and tumor-node-metastasis (TNM) stage (Table 1). In the glycolysis-low group, 42 (35.2\%) patients were HPV-positive, whereas in the glycolysis-high group, 55 (13.7\%) patients were HPV-positive. Regarding primary lesions, the proportion of oral cavity lesions was higher in the glycolysis-high group (71.1\%) than in the glycolysis-low group (55.4\%); however, the proportion of oropharynx lesions was lower in the glycolysis-high group (5.5\%) than in the glycolysis-low group (17.6\%). In the glycolysis-low group, the proportion of patients with Tis, T1, and T2 tumors (51.3\%) was higher than that of patients with T3 and T4 tumors (48.7\%). However, in the glycolysis-high group, the proportion of patients with Tis, $\mathrm{T} 1$, and T2 tumors was lower $(37.2 \%)$ than that of patients with T3 and T4 tumors (62.8\%). No differences in the $\mathrm{N}$ factor, $\mathrm{M}$ factor, and TNM stage were observed between the groups. Additionally, we performed survival analyses to compare survival between the groups (Figure 
1b). The glycolysis-high group exhibited shorter overall survival (OS) than the glycolysis-low group, especially in patients who were HPV-positive. Additionally, the glycolysis-high group exhibited shorter disease-free survival than the glycolysis-low group.

\section{Glycolysis-high gene signature correlated with various hallmark pathways}

To investigate the biological differences between the glycolysis-low and glycolysis-high groups, we compared the groups and identified 2366 differentially expressed genes (DEGs), including 459 upregulated and 1907 downregulated genes in the glycolysis-high group (Figure 2a, Suppl. Table 1). To explore the biological functions of DEGs and related pathways, we conducted Gene set enrichment analysis (GSEA; Figure 2b, 2c). In the glycolysis-high group, 10 hallmark pathways were upregulated and two pathways were downregulated (FDR< 0.10$)$. Various pathways representing cancer hallmarks, such as interferon-alpha (IFN-a) response, myc targets, hypoxia, epithelial-mesenchymal transition (EMT), transforming growth factor (TGF)- $\beta$ signaling, and interleukin 6 (IL6)-Janus kinase (JAK)-signal transducer and activator of transcription 3 (STAT3) signaling, were upregulated in the glycolysis-high group.

\section{Correlation between glycolysis activity and immune cell infiltration}

We next calculated the enrichment scores of 16 basic immune cell types to assess

the correlation between glycolysis activity and immune cell infiltration. The immune score and the enrichment scores of CD4+ T cells, CD8+ T cells (not significant), B cells, and dendritic cells (DCs) were lower in the glycolysis-high group than in the glycolysis-low group. However, the enrichment scores of Th2 cells, NKT cells, monocytes, and neutrophils were higher than those in the glycolysis-low group than in the glycolysis-high group (Figure 3a). We also examined the correlation between each glycolysis gene and immune cell enrichment scores, which indicated the same trends (Figure $3 \mathrm{~b}$ ).

\section{Glycolysis activity correlated with the immunosuppressive microenvironment}

We compared the expression of immune-related genes to emphasize immunological differences between glycolysis groups (Figure 4a). The expression of immunosuppressive genes, including TGFB1, CD274, and $P D C D 1 L G 2$, was higher in the glycolysis-high group than in the glycolysis-low group (Figure 4b). In contrast, the expression of immune checkpoint molecules, including CTLA4, PDCD1, and TIGIT, was lower in the glycolysis-high group than in the glycolysis-low group (Figure 4b). No difference in the expression of the immunostimulatory genes IFNG and GZMB was observed between glycolysis groups (Suppl. Figure 1). Similar trends were observed between each glycolysis gene and immune-related genes (Figure 4c).

\section{Discussion}

Altered metabolism is an emerging hallmark of cancer ${ }^{10,11}$. Various cancers exhibit upregulated glycolysis and downregulated mitochondrial oxidative phosphorylation. Immune evasion has recently 
attracted attention due to recent advances in cancer immunotherapy. In the present study, we performed a comprehensive analysis of the transcriptome and clinical data regarding HNSCC obtained from TCGA database. The glycolysis-high signature positively correlated with HPV-negative tumor type, advanced T factor, and unfavorable prognosis. GSEA revealed the upregulation of various pathways representing cancer hallmarks in the glycolysis-high group. Immune cell enrichment analyses showed decreased infiltration of T cells, DCs, and B cells and increased infiltration of monocyte and neutrophil in the glycolysis-high group. Moreover, the expression of immunosuppressive genes, including TGFB1, CD274, and PDCD1LG2, was upregulated in the glycolysis-high group. These results suggest the potential of monitoring glycolysis as a biomarker for tumor progression and immunological status in the TME of HNSCC.

Non-supervised hierarchical clustering analysis revealed a positive correlation between glycolysis and advanced T factors. In normal cells, mitochondrial oxidative phosphorylation is widely used to efficiently generate energy. However, cancer cells often rely on aerobic glycolysis, resulting in remarkably upregulated glycolysis, although not an efficient strategy to produce adenosine 5'-triphosphate (ATP). Accelerated cell proliferation has been implicated in the dependence of cancer cells on glycolysis. For rapid proliferation, cells require nutrient metabolism to generate nucleotides, amino acids, and lipids rather than efficient ATP production. In cancer cells, upregulated glycolysis results from altered metabolic pathways that provide biomass for cell proliferation ${ }^{12,29}$. Consequently, the advanced $T$ factor in the glycolysis-high group could be attributed to accelerated cell proliferation, which relies on aerobic glycolysis. Furthermore, we observed a positive correlation between the HPV-negative tumor type and the glycolysis-high signature. Comprehensive analysis of the TCGA database indicated frequent alterations in TP53 and CDKN2A in HPV-negative HNSCC, but not in HPV-positive HNSCC. TP53 is a negative regulator of glycolysis ${ }^{30}$. Therefore, upregulated glycolysis may be a biological feature of HPV-negative carcinogenesis. In addition, upregulated glycolysis is an unfavorable prognostic factor. Upregulation of glycolysis in cancer cells results in the accumulation of lactate in the $\mathrm{TME}^{12}$. A high lactic acid concentration in the TME inhibits the cytotoxic activity of cytotoxic T cells, resulting in immune evasion of tumors ${ }^{31}$. A lactate-enriched TME also mediates increased invasion, apoptosis resistance, and increased survival and proliferation of tumor cells ${ }^{32-34}$. Therefore, advanced T factor and a lactateenriched TME may be associated with shorter survival in glycolysis-upregulated HNSCCs.

We further compared the two glycolysis groups using GSEA. Various pathways that represent cancer hallmarks were upregulated in the glycolysis-high group. The IFN-a response pathway exhibited the highest normalized enrichment score. IFN- $a$ is a cytokine family that belongs to type I IFNs ${ }^{35}$. Based on its stimulatory effects on innate and adaptive immunity, IFN-a has been widely used in clinical oncology to treat various cancer types, especially hematological malignancies ${ }^{36}$. However, recent studies have demonstrated the pro-tumoral role of IFN-a signaling. In HNSCC, IFN-a signaling promotes the immunosuppressive TME by activating the PD-1-programmed death ligand-1 (PD-L1) axis ${ }^{37}$. Therefore, the upregulation of PD-1 ligand genes, CD274 and PDCD1LG2, in the glycolysis-high group could be related to the upregulated IFN-a response. Although the abundance of IFN- $y$ in the TME has been 
recognized as a key mechanism that induces PD-L1 expression in tumor cells ${ }^{38}$, our results suggest that IFN- $a$ could also be an important mechanism for PD-L1 induction in glycolysis-upregulated HNSCC. Moreover, growing evidence suggests that IFN-a signaling contributes to increased migration, drug resistance, and immune evasion in breast cancer ${ }^{39}$. Further investigations focused on the relationship between IFN-a signaling and glycolysis in the TME of HNSCC are needed. Furthermore, GSEA revealed that that the EMT pathway and TGF- $\beta$ signaling pathway were upregulated in the glycolysis-high signature. EMT is a process in which cells lose their epithelial features and acquire mesenchymal traits, including motility, invasiveness, and various characteristics of stem cells ${ }^{40}$. TGF- $\beta$ is a well-known cytokine family and a major driver of $\mathrm{EMT}^{41}$. Accumulating evidence suggests that TGF- $\beta$ signaling also enhances glycolysis by activating various glycolytic enzymes, including GLUT1, hexokinase 2, 6phosphofructo-2-kinase/fructose-2,6-biphosphatase 3, 6-phosphofructo-1-kinase, pyruvate kinase M2, and lactate dehydrogenase type $A^{42}$. Consistent with these findings, TGF- $\beta$ signaling may play a vital role in the induction of both glycolysis and EMT in HNSCC. Additionally, the significant upregulation of myc target pathways and hypoxia signaling was consistent with their roles in the induction of glycolysis ${ }^{20,24-}$ 26 .

Immune cell enrichment analysis revealed a negative correlation between glycolysis and infiltration of CD4+ $T$ cells, $C D 8+T$ cells, $B$ cells, and DCs. The interaction between DCs and $T$ cells is an important axis of the anti-tumor immune response through tumor-antigen presentation and $\mathrm{T}$ cell activation ${ }^{43}$. The negative effects of the lactate-enriched TME on T cell proliferation, cytotoxic activity of T cells, and maturation of DCs support our observation ${ }^{44,45}$. Moreover, the comparison of immune-related gene expression between the glycolysis groups revealed higher expressions of CD274, PDCD1LG2, and TGFB1 in the glycolysis-high group, which facilitated T cell exhaustion and dysfunction in the TME ${ }^{46}$. Therefore, the decreased infiltration of T cells and DCs in the glycolysis-high group may be related to increased immune evasion in the glycolysis-elevated TME. The lower gene expression of immune checkpoint molecules on T cells, such as $C T L A 4, P D C D 1$, and $T I G I T$, may reflect lower T cell infiltration in the glycolysis-high group.

Recent advances in cancer immunotherapy targeting solid tumors have mainly focused on T cells; however, the anti-tumor roles of B cells through antigen presentation and antibody production have been recently recognized ${ }^{47}$. The significant decrease in $\mathrm{B}$ cell infiltration in the glycolysis-high group may also reflect immune evasion in the TME of HNSCC. Moreover, both monocytes and neutrophils represent diverse functions and subsets, facilitating pro-tumoral and anti-tumoral immune responses ${ }^{48,49}$. Although increased infiltration of both monocytes and neutrophils in the glycolysis-high group could be an aspect of immune evasion in the glycolysis-elevated TME, further characterization of these immune cell types would be necessary.

There are limitations to the present study. We performed a comprehensive analysis using bulk RNA sequencing data. In the case of bulk RNA sequencing, gene expression values represent not only mRNA expression in tumor cells but also that in all other cell types existing in the TME. This method should work 
well for genes that are specific to certain cell types; however, for genes that are commonly expressed in various cell types, single-cell RNA sequencing would be better suited for a more precise analysis of the TME. Accumulation of public archives of single-cell RNA sequencing data is needed for an in-depth characterization of the metabolic state in the TME.

In conclusion, we elucidated the correlation between upregulated glycolysis and several clinical and transcriptomic significances in HNSCC by analyzing data from TCGA database. Glycolysis monitoring has the potential as a biomarker for tumor progression and immunological status in the TME of HNSCC.

\section{Materials And Methods}

\section{TCGA data}

RNA-sequencing data (Illumina Hiseq RNAseq V2, raw counts, and FPKM values) and clinical information were obtained from TCGA Research Network (TCGA Provisional version updated in 2016, http://cancergenome.nih.gov/). Data on 520 patients, including 97 patients with HPV-positive HNSCC and 423 patients with HPV-negative HNSCC, were analyzed. The $\log _{10}$-transformed values of mRNA expression levels were calculated.

\section{Hierarchical clustering of patients with glycolysis-related genes}

All patients with HNSCC underwent non-supervised hierarchical clustering based on z-scores of the expression of 16 glycolysis-related genes. The following 16 glycolysis-related genes were used based on the glycolysis core pathways of the Reactome pathway database: PFKP, PGK1, PGAM1, PGAM4, ENO1, PKM2, LDHA, TPI1, GAPDH, GPI, ALDOA, HK1, SLC2A1, HK2, ALDOC, and PKLR ${ }^{50}$. Patients were divided into two groups-the glycolysis-low and glycolysis-high groups-using the cutree $\mathrm{R}$ function based on clustering results. The heat map was illustrated using the pheatmap R package.

\section{Differentially expressed gene analysis}

In the glycolysis-low and glycolysis-high groups, DEGs were identified using the ExperimentHub R package and DESeq2 R package. DEGs were filtered using the threshold $\left|\log _{2} \mathrm{FC}\right| \geq 1$ and adjusted $P$-value of $<0.05$. A volcano plot was used to visualize $D E G s$ using the calibration $R$ package.

\section{Gene set enrichment analysis}

GSEA (GSEA v4, Broad Institute) was performed to identify pathways upregulated in the glycolysis-high group. The normalized enrichment score, $P$-value, and false discovery rate (FDR) q-value were calculated for each gene set using the Hallmark pathway database. A correlation plot was constructed using the corrplot and pheatmap R packages.

\section{Cell type enrichment analysis}


The xCell tool, a gene signature-based method, was employed to evaluate the enrichment of various cell types in 520 HNSCC tissues. Among the 64 cell types, the enrichment scores of 13 basic immune cell types were calculated using the xCell $\mathrm{R}$ package.

\section{Statistical analysis}

Data were analyzed using R (version 4.0.3; The R Foundation for Statistical Computing, Vienna, Austria)

in combination with R studio version 1.3.1093 (R studio, Boston, MA, USA) and GraphPad Prism version 8 (GraphPad Software, San Diego, CA, USA). Student's t-test was used to compare continuous variables between the groups. The chi-square test for independence and Fisher's exact test were used to compare categorical variables. Pearson's correlation coefficient was utilized to evaluate the correlation between two continuous variables. Two-sided $P$-values of $<0.05$ were considered statistically significant. Survival curves were calculated using the Kaplan-Meier method and compared using the log-rank test.

\section{Declarations}

\section{Acknowledgments}

This work was supported in part by a Grant-in-Aid for Scientific Research (B) $20 \mathrm{H} 03834$ (K.C.) and Grantin-Aid for Young Scientists 20K18243 (H.T.) from the Ministry of Education, Culture, Sports, Science and Technology, Japan.

\section{Author contributions}

HT, Conceptualization, Methodology, Validation, Investigation, Resources, Writing - original draft preparation, Project administration, Funding acquisition; RK, Methodology, Resources, Writing - review and editing, Visualization; SI, Software, Formal analysis; IM, Software, Formal analysis; HT, Software, Formal analysis; KC, Writing - review and editing, Supervision, Funding acquisition.

\section{Additional information}

Disclosure Statement: The authors declare no potential conflicts of interest.

\section{References}

1 Ferlay, J. et al. Estimating the global cancer incidence and mortality in 2018: GLOBOCAN sources and methods. Int J Cancer144, 1941-1953, doi:10.1002/ijc.31937 (2019).

2 Bray, F. et al. Global cancer statistics 2018: GLOBOCAN estimates of incidence and mortality worldwide for 36 cancers in 185 countries. CA Cancer J Clin68, 394-424, doi:10.3322/caac.21492 (2018).

3 Hashibe, M. et al. Alcohol drinking in never users of tobacco, cigarette smoking in never drinkers, and the risk of head and neck cancer: pooled analysis in the International Head and Neck Cancer 
Epidemiology Consortium. J Nat/ Cancer Inst99, 777-789, doi:10.1093/jnci/djk179 (2007).

4 Mehanna, H. et al. Prevalence of human papillomavirus in oropharyngeal and nonoropharyngeal head and neck cancer--systematic review and meta-analysis of trends by time and region. Head Neck35, 747755, doi:10.1002/hed.22015 (2013).

5 Ferris, R. L. et al. Nivolumab for Recurrent Squamous-Cell Carcinoma of the Head and Neck. N Engl J Med375, 1856-1867, doi:10.1056/NEJMoa1602252 (2016).

6 Burtness, B. et al. Pembrolizumab alone or with chemotherapy versus cetuximab with chemotherapy for recurrent or metastatic squamous cell carcinoma of the head and neck (KEYNOTE-048): a randomised, open-label, phase 3 study. Lancet394, 1915-1928, doi:10.1016/S0140-6736(19)32591-7 (2019).

7 Tong, C. C., Kao, J. \& Sikora, A. G. Recognizing and reversing the immunosuppressive tumor microenvironment of head and neck cancer. Immunol Res54, 266-274, doi:10.1007/s12026-012-8306-6 (2012).

8 Chen, S. M. Y. et al. Tumor immune microenvironment in head and neck cancers. Mol Carcinog, doi:10.1002/mc.23162 (2020).

9 Seliger, B. Strategies of tumor immune evasion. BioDrugs19, 347-354, doi:10.2165/00063030200519060-00002 (2005).

10 Hanahan, D. \& Weinberg, R. A. Hallmarks of cancer: the next generation. Cel/144, 646-674, doi:10.1016/j.cell.2011.02.013 (2011).

11 Hanahan, D. \& Weinberg, R. A. The hallmarks of cancer. Cel/100, 57-70, doi:10.1016/s00928674(00)81683-9 (2000).

12 Vander Heiden, M. G., Cantley, L. C. \& Thompson, C. B. Understanding the Warburg effect: the metabolic requirements of cell proliferation. Science324, 1029-1033, doi:10.1126/science.1160809 (2009).

13 Warburg, O., Wind, F. \& Negelein, E. THE METABOLISM OF TUMORS IN THE BODY. J Gen Physio/8, 519-530, doi:10.1085/jgp.8.6.519 (1927).

14 Bonomo, P. et al. What is the prognostic impact of FDG PET in locally advanced head and neck squamous cell carcinoma treated with concomitant chemo-radiotherapy? A systematic review and metaanalysis. Eur J Nucl Med Mol Imaging45, 2122-2138, doi:10.1007/s00259-018-4065-5 (2018).

15 Cascone, T. et al. Increased Tumor Glycolysis Characterizes Immune Resistance to Adoptive T Cell Therapy. Cell Metab27, 977-987.e974, doi:10.1016/j.cmet.2018.02.024 (2018). 
$16 \mathrm{Li}, \mathrm{Z}$. \& Zhang, H. Reprogramming of glucose, fatty acid and amino acid metabolism for cancer progression. Cell Mol Life Scl73, 377-392, doi:10.1007/s00018-015-2070-4 (2016).

17 Sattler, U. G. et al. Glycolytic metabolism and tumour response to fractionated irradiation. Radiother Onco/94, 102-109, doi:10.1016/j.radonc.2009.11.007 (2010).

18 Carvalho, K. C. et al. GLUT1 expression in malignant tumors and its use as an immunodiagnostic marker. Clinics (Sao Paulo)66, 965-972, doi:10.1590/s1807-59322011000600008 (2011).

19 Kimmelman, A. C. Metabolic Dependencies in RAS-Driven Cancers. Clin Cancer Res21, 1828-1834, doi:10.1158/1078-0432.CCR-14-2425 (2015).

20 Tateishi, K. et al. Myc-Driven Glycolysis Is a Therapeutic Target in Glioblastoma. Clin Cancer Res22, 4452-4465, doi:10.1158/1078-0432.CCR-15-2274 (2016).

21 Liu, Q. P., Luo, Q., Deng, B., Ju, Y. \& Song, G. B. Stiffer Matrix Accelerates Migration of Hepatocellular Carcinoma Cells through Enhanced Aerobic Glycolysis Via the MAPK-YAP Signaling. Cancers (Basel)12, doi:10.3390/cancers12020490 (2020).

$22 \mathrm{Xie}$, Y. et al. PI3K/Akt signaling transduction pathway, erythropoiesis and glycolysis in hypoxia (Review). Mol Med Rep19, 783-791, doi:10.3892/mmr.2018.9713 (2019).

23 Muller, P. A. \& Vousden, K. H. p53 mutations in cancer. Nat Cell Bio/15, 2-8, doi:10.1038/ncb2641 (2013).

24 DeBerardinis, R. J., Lum, J. J., Hatzivassiliou, G. \& Thompson, C. B. The biology of cancer: metabolic reprogramming fuels cell growth and proliferation. Cell Metab7, 11-20, doi:10.1016/j.cmet.2007.10.002 (2008).

25 Semenza, G. L. HIF-1: upstream and downstream of cancer metabolism. Curr Opin Genet Dev20, 51-56, doi:10.1016/j.gde.2009.10.009 (2010).

26 Jones, R. G. \& Thompson, C. B. Tumor suppressors and cell metabolism: a recipe for cancer growth. Genes Dev23, 537-548, doi:10.1101/gad.1756509 (2009).

27 Chang, C. H. et al. Metabolic Competition in the Tumor Microenvironment Is a Driver of Cancer Progression. Cel/162, 1229-1241, doi:10.1016/j.cell.2015.08.016 (2015).

28 Justus, C. R., Sanderlin, E. J. \& Yang, L. V. Molecular Connections between Cancer Cell Metabolism and the Tumor Microenvironment. Int J Mol Sci16, 11055-11086, doi:10.3390/ijms160511055 (2015).

29 Salazar-Roa, M. \& Malumbres, M. Fueling the Cell Division Cycle. Trends Cell Bio/27, 69-81, doi:10.1016/j.tcb.2016.08.009 (2017). 
30 Eriksson, M. et al. Effect of Mutant p53 Proteins on Glycolysis and Mitochondrial Metabolism. Mol Cell Bio/37, doi:10.1128/MCB.00328-17 (2017).

31 Fischer, K. et al. Inhibitory effect of tumor cell-derived lactic acid on human T cells. Blood109, 38123819, doi:10.1182/blood-2006-07-035972 (2007).

32 Doherty, J. R. \& Cleveland, J. L. Targeting lactate metabolism for cancer therapeutics. J Clin Invest123, 3685-3692, doi:10.1172/JCl69741 (2013).

33 Hirschhaeuser, F., Sattler, U. G. \& Mueller-Klieser, W. Lactate: a metabolic key player in cancer. Cancer Res71, 6921-6925, doi:10.1158/0008-5472.CAN-11-1457 (2011).

34 Gatenby, R. A., Gawlinski, E. T., Gmitro, A. F., Kaylor, B. \& Gillies, R. J. Acid-mediated tumor invasion: a multidisciplinary study. Cancer Res66, 5216-5223, doi:10.1158/0008-5472.CAN-05-4193 (2006).

35 Rizza, P., Moretti, F. \& Belardelli, F. Recent advances on the immunomodulatory effects of IFN-alpha: implications for cancer immunotherapy and autoimmunity. Autoimmunity43, 204-209, doi:10.3109/08916930903510880 (2010).

36 Ferrantini, M., Capone, I. \& Belardelli, F. Interferon-alpha and cancer: mechanisms of action and new perspectives of clinical use. Biochimie89, 884-893, doi:10.1016/j.biochi.2007.04.006 (2007).

$37 \mathrm{Ma}, \mathrm{H}$. et al. Interferon-alpha promotes immunosuppression through IFNAR1/STAT1 signalling in head and neck squamous cell carcinoma. Br J Cancer120, 317-330, doi:10.1038/s41416-018-0352-y (2019).

38 Shi, Y. Regulatory mechanisms of PD-L1 expression in cancer cells. Cancer Immunol Immunother67, 1481-1489, doi:10.1007/s00262-018-2226-9 (2018).

39 Provance, O. K. \& Lewis-Wambi, J. Deciphering the role of interferon alpha signaling and microenvironment crosstalk in inflammatory breast cancer. Breast Cancer Res21, 59, doi:10.1186/s13058-019-1140-1 (2019).

40 Thiery, J. P., Acloque, H., Huang, R. Y. \& Nieto, M. A. Epithelial-mesenchymal transitions in development and disease. Cel/139, 871-890, doi:10.1016/j.cell.2009.11.007 (2009).

41 Inman, G. J. Switching TGF $\beta$ from a tumor suppressor to a tumor promoter. Curr Opin Genet Dev21, 9399, doi:10.1016/j.gde.2010.12.004 (2011).

42 Hua, W., Ten Dijke, P., Kostidis, S., Giera, M. \& Hornsveld, M. TGFß-induced metabolic reprogramming during epithelial-to-mesenchymal transition in cancer. Cell Mol Life Scl77, 2103-2123, doi:10.1007/s00018-019-03398-6 (2020).

43 Wculek, S. K. et al. Dendritic cells in cancer immunology and immunotherapy. Nat Rev Immuno/20, 724, doi:10.1038/s41577-019-0210-z (2020). 
44 Deberardinis, R. J., Sayed, N., Ditsworth, D. \& Thompson, C. B. Brick by brick: metabolism and tumor cell growth. Curr Opin Genet Dev18, 54-61, doi:10.1016/j.gde.2008.02.003 (2008).

45 Gottfried, E. et al. Tumor-derived lactic acid modulates dendritic cell activation and antigen expression. Blood107, 2013-2021, doi:10.1182/blood-2005-05-1795 (2006).

46 Thommen, D. S. \& Schumacher, T. N. T Cell Dysfunction in Cancer. Cancer Cel/33, 547-562, doi:10.1016/j.ccell.2018.03.012 (2018).

47 Fridman, W. H. et al. B cells and cancer: To B or not to B? J Exp Med218, doi:10.1084/jem.20200851 (2021).

48 Jaillon, S. et al. Neutrophil diversity and plasticity in tumour progression and therapy. Nat Rev Cancer20, 485-503, doi:10.1038/s41568-020-0281-y (2020).

49 Olingy, C. E., Dinh, H. Q. \& Hedrick, C. C. Monocyte heterogeneity and functions in cancer. J Leukoc Bio/106, 309-322, doi:10.1002/JLB.4RI0818-311R (2019).

50 Fabregat, A. et al. Reactome pathway analysis: a high-performance in-memory approach. BMC Bioinformatics18, 142, doi:10.1186/s12859-017-1559-2 (2017).

\section{Tables}


Table 1. Relationship between glycolysis activity and clinical parameters in 520 patients with HNSCC

Variables Glycolysis

$\underset{(n=119)}{\text { Low }} \quad \stackrel{\text { High }}{(n=401)} \quad$ P-value

HPV status

$\begin{array}{llll}\text { Negative } & 77 & 346 & <0.0001 \\ \text { Positive } & 42 & 55 & \end{array}$

Primary lesion

Hypopharynx

2

8

0.0001

Larynx

30

86

Oral cavity

66

285

Oropharynx

21

22

$T$ factor

Tis-2

61

149

0.006

T3-4

58

252

$\mathrm{N}$ factor

Negative

41

172

0.12

Positive

70

208

M factor

Negative

116

388

0.22

Positive

0

5

TNM stage

$\begin{array}{llll}\text { I-II } & 25 & 86 & 0.92 \\ \text { III-IV } & 94 & 315 & \end{array}$

Abbreviations: HNSCC, head and neck squamous cell carcinoma; HPV, human papillomavirus; TNM, tumor-node-metastasis.

Figures 
(a)

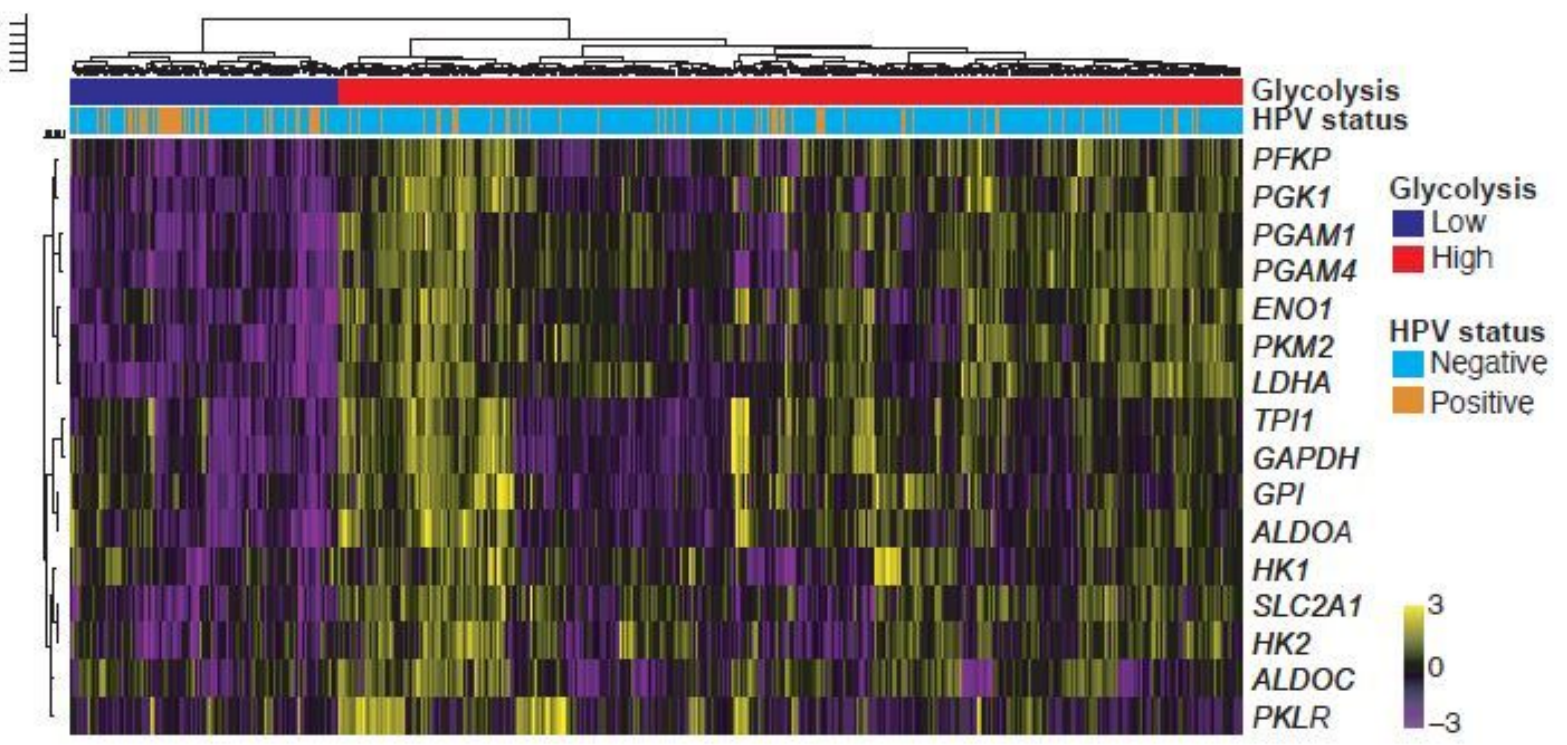

(b)
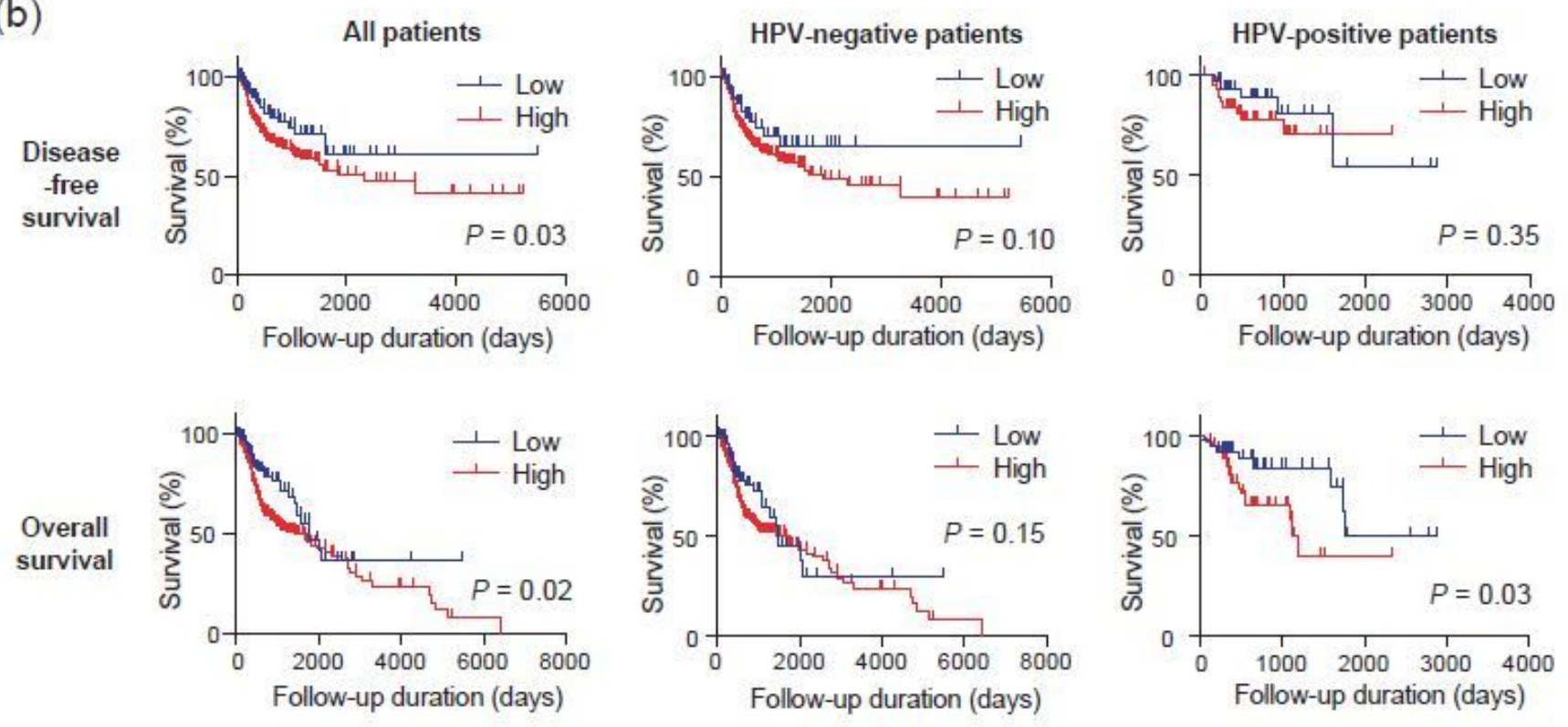

\section{Figure 1}

Glycolysis-high gene signature positively correlated with shorter survival a-b, mRNA expression data of 16 glycolysis-related genes and clinical information were obtained from TCGA database. a, Heat map of glycolysis-related gene expression in 520 patients with head neck squamous cell carcinoma (HNSCC). All patients with HNSCC underwent non-supervised hierarchical clustering based on the z-scores of $\log 10-$ transformed expressions. b, Kaplan-Meier survival curves based on glycolysis groups. Disease-free survival was evaluated in all patients $(n=429)$, HPV-negative patients $(n=348)$, and HPV-positive patients $(n=81)$. Overall survival was evaluated in all patients $(n=495)$, HPV-negative patients $(n=403)$, and HPV-positive patients $(n=92)$. TCGA, The Cancer Genome Atlas; HPV, human papillomavirus. 
(a)

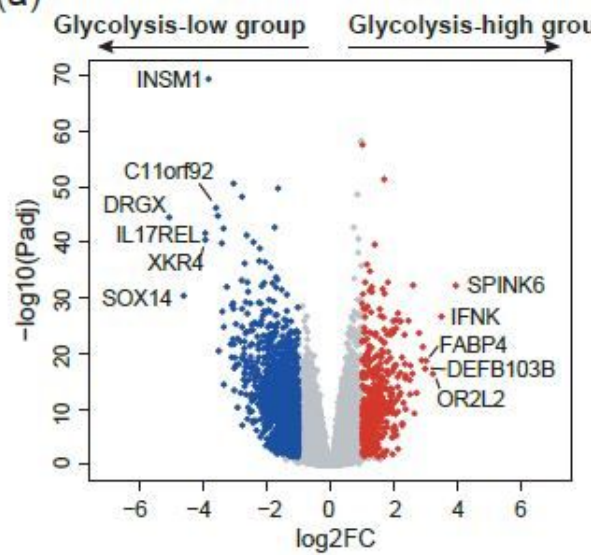

(b)

Hallmark pathways Normalized enrichment score

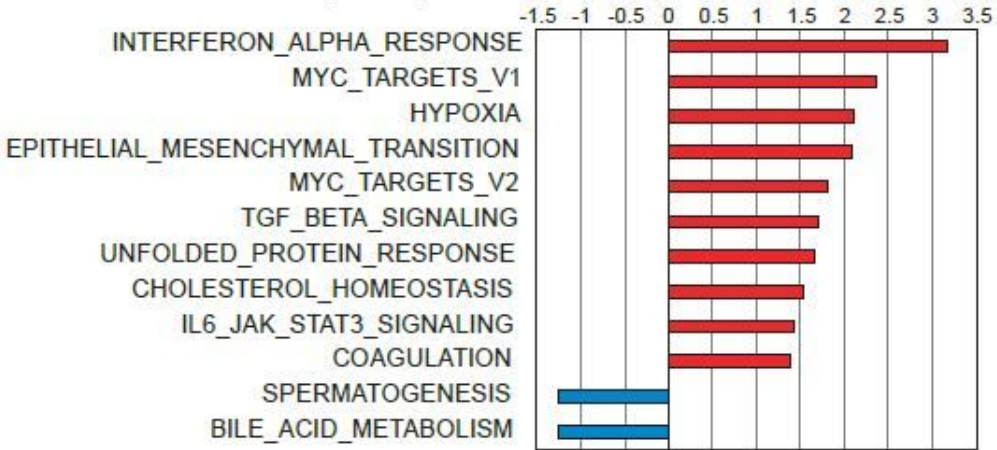

(c)
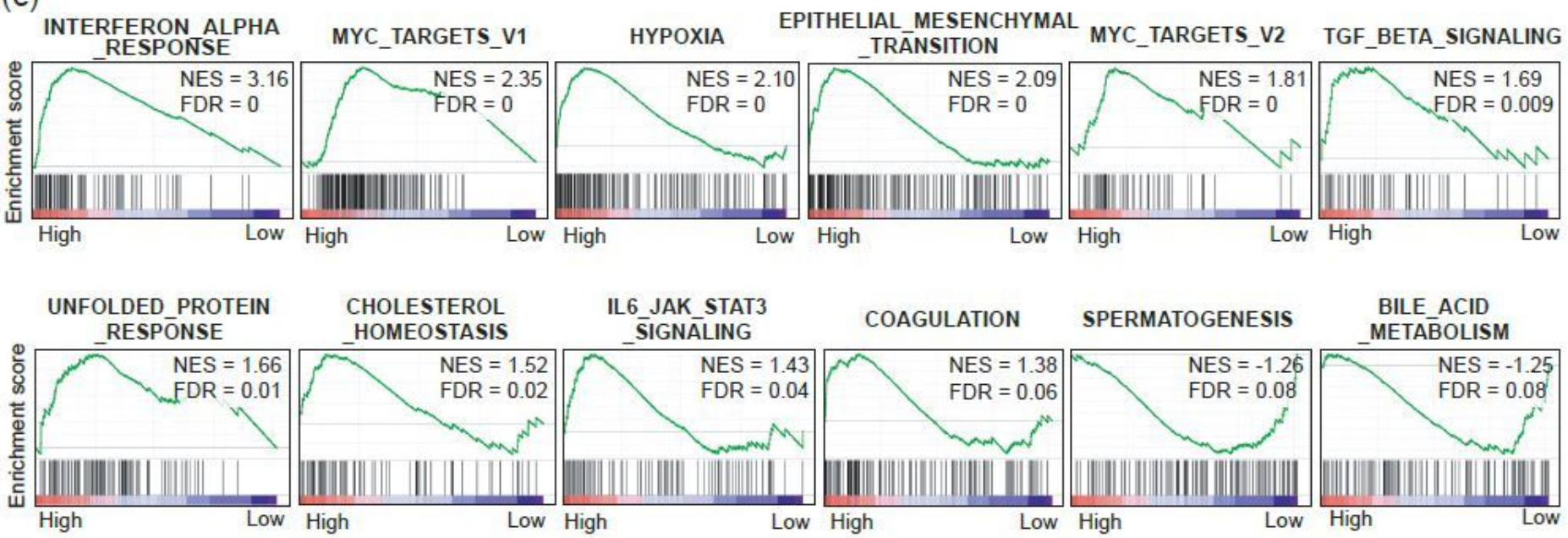

Figure 2

Differentially expressed genes and upregulated pathways in the glycolysis-high group a, Volcano plot of differentially expressed genes in the glycolysis-high group. Red dots represent upregulated genes (Padj < $0.05, \log 2 \mathrm{FC}>1)$, whereas blue dots represent downregulated genes (Padj $<0.05, \log 2 \mathrm{FC}<-1) . \mathrm{b}$, Upregulated and downregulated hallmark pathways in the glycolysis-high group obtained by GSEA (FDR $<0.10)$. c, GSEA plots of pathways shown in b. Padj, adjusted P-value; GSEA, gene set enrichment analysis. 
(a)
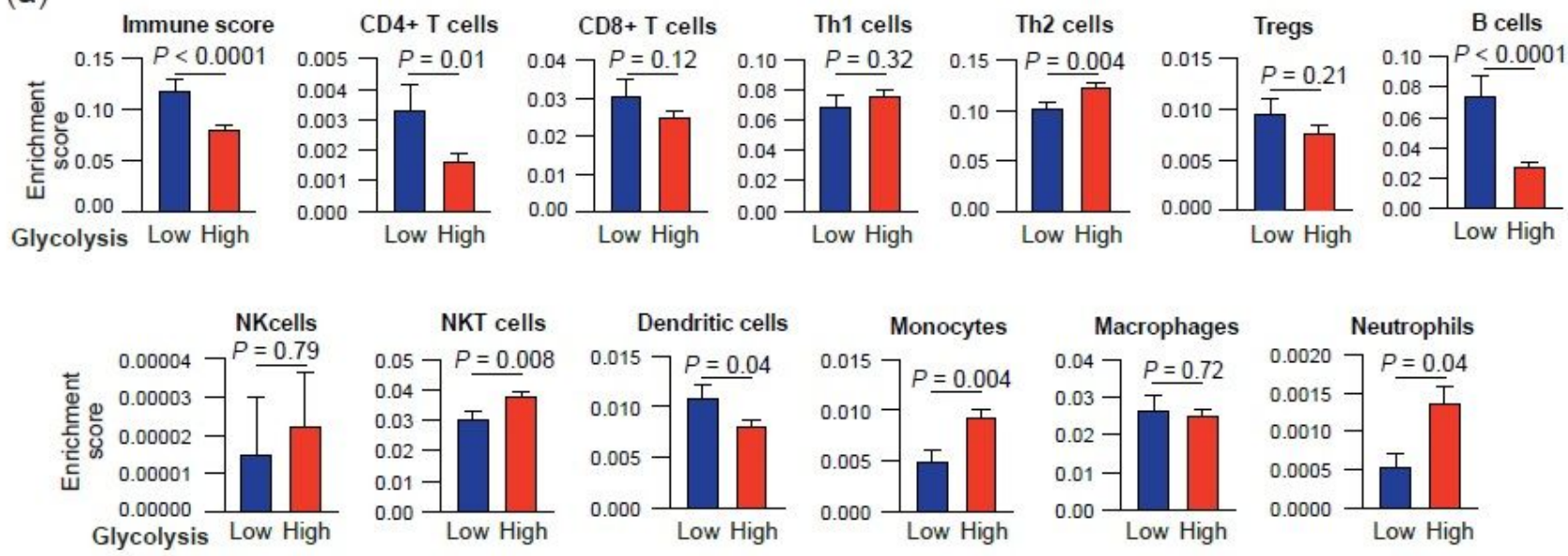

(b)

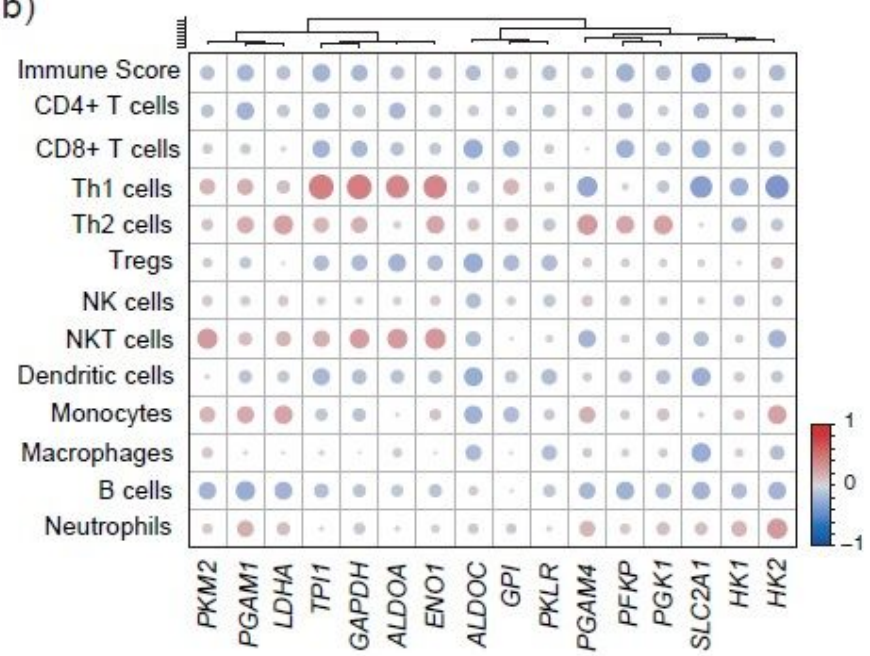

Figure 3

Correlation between glycolysis activity and immune cell enrichment scores a, Bar graphs of immune cell enrichment scores in the glycolysis-high and glycolysis-low groups. b, Correlation matrix displaying Rvalues for assessing the correlation between the expression of 16 glycolysis-related genes and immune cell enrichment scores across 520 HNSCC tissues. The glycolysis-related genes were clustered by hierarchical clustering based on R values. HNSCC, head neck squamous cell carcinoma. 
(a)

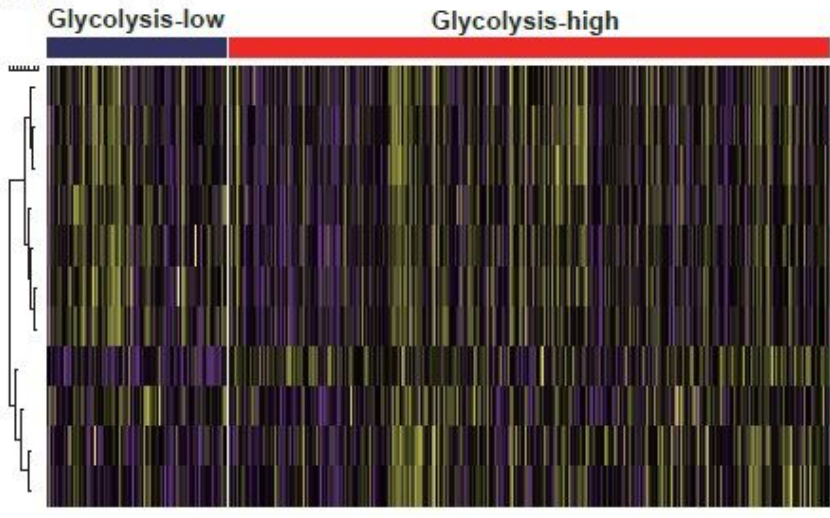

IFNG

GZMB

LAG3

HAVCR2

CTLA4 *

PDCD1 *

TIGIT **

TGFB1 $1^{* * \star \star}$

L10

CD274 ****

PDCD1LG2 **** (b)
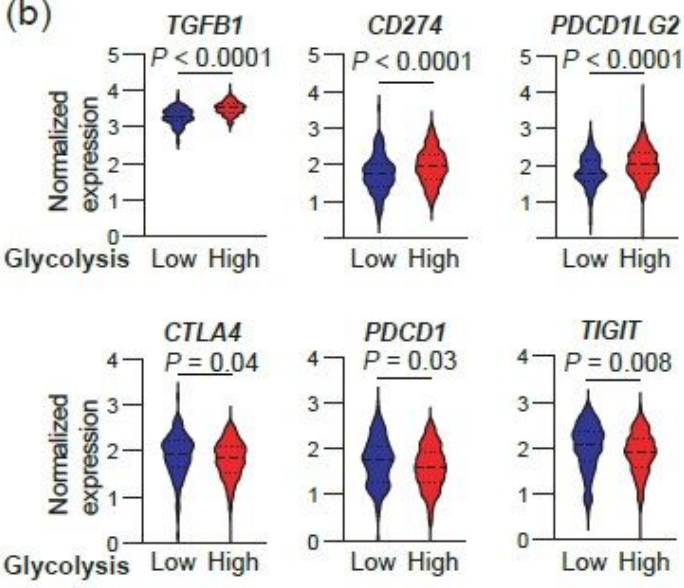

(c)

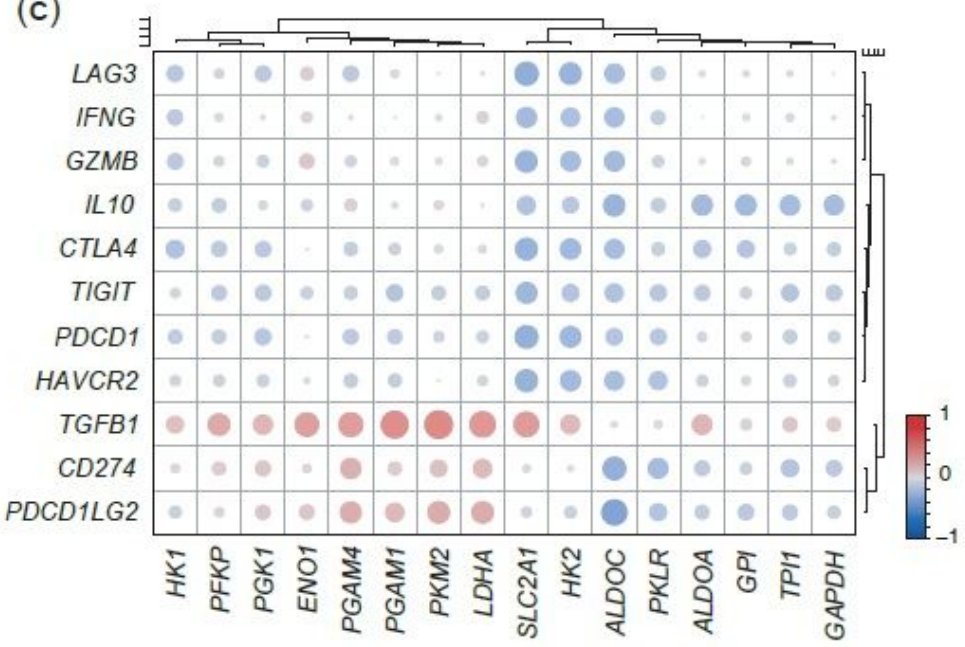

\section{Figure 4}

Correlation between glycolysis activity immune-related genes a, Heat map of immune-related gene expression in the glycolysis-high and glycolysis-low groups. b, Violin plots of normalized gene expressions that were significantly upregulated or downregulated in the glycolysis-high group shown in a. c, Correlation matrix displaying R-values for assessing the correlation between the expression of 16 glycolysis-related genes and that of immune-related genes across 520 HNSCC tissues. Glycolysis-related genes and immune-related genes were clustered by hierarchical clustering based on the R values. ${ }^{*} \mathrm{P}<$ $0.05 ; * \star, P<0.01 ; * \star \star, P<0.001 ; \star \star \star \star, P<0.0001$. HNSCC, head neck squamous cell carcinoma.

\section{Supplementary Files}

This is a list of supplementary files associated with this preprint. Click to download.

- TakahashietalSuppl.Table1.xlsx

- GlycolysisSupple.Figure1.pdf 